Canadian Journal of Family and Youth, 5(1), 2013, pp 91-106

ISSN 1718-9748 @ University of Alberta

http://ejournals.library.ualberta.ca/index/php/cjfy

\title{
A Review of the Literature on Sexual Development of Older Adults in Relation to the Asexual Stereotype of Older Adults
}

\author{
Róisín T. Kenny
}

\begin{abstract}
There is a prevalence of an asexual stereotype in regard to older adults. This paper reviews sexual development later in life from three different vantage points to explore why and how this stereotype exists. The vantage points are changes in social life, physical development, and psychological development. Older adults are often less engaged in society due to retirement, hence they have fewer opportunities to meet new partners. However now the internet is used a forum for older adults to meet new partners, so social barriers are easily overcome. Furthermore, the pervasive social influence of the media, reinforces and perpetuates the asexual stereotype in society by narrowly portraying older adults as asexual. Normative physical changes that come with aging, such as menopause and erectile dysfunction, are frequently assumed to lead to asexuality. This is not necessarily true- those that see physical changes as natural adapt their sexual lives accordingly. Psychological factors that effect sexuality in later life are the internalization of societies prescription of what 'normal' sex life is for older adults. As a result it is common for older adults to deprioritize sex as a means to conform to 'norms' regarding sex later in life.
\end{abstract}

Róisín Kenny is currently completing an undergraduate degree in Psychology at Grant MacEwan University. When not engaged in academic pursuits, Róisín works as a private contractor for the Truth and Reconciliation Commission of Canada. She won first place in MacEwan's 2012 academic conference, 'Domination', for her presentation on the concept of reconciliation. Other academic achievements include publication in MacEwan's undergraduate journal Confluence, as well as having completed a clinical psychology field placement at the Grey Nuns Hospital. Upon graduation, Róisín intends to continue her education in psychology at the graduate level. 
Kenny

\section{Introduction}

Sexual development from birth to the end of the fertile period is a well-researched subject in the social sciences, as it is thought to be of importance to the human experience. Sexual development of older people, however, has received much less attention; this is due to the widely held belief that older adults are asexual. Research shows that the sexual health of older people is frequently ignored at a policy level, as evident in studies conducted on sexual health that place an upper limit on participants such as the National Survey of Sexual Attitudes (Gott \& Hinchliff, 2003). Research studies rooted in ageist beliefs of the asexuality of older people have resulted in the absence of a comprehensive body of knowledge on sexuality of older people. The research that does exist on the sexuality of older adults asserts that sexuality generally does continue to have importance in later life (Gott \& Hinchliff, 2003). In fact, one study found that out of those participants (aged 70 to 80) that had sexual intercourse within the past year, almost a quarter of them had intercourse more frequently than once a week (Hillman, 2008). It is estimated that the entire cohort of people over age 65 will more than double in size by year 2030 (Hillman, 2008). Hence, knowledge about sexual health of the older demographic is going to be of greater and greater importance in the next 20 years as we experience the graying of the population.

In this paper I plan to review scholarly literature on sexual development of older people in relation to the stereotype of asexuality of the older population. In doing so, I hope to demonstrate the invalidity of the stereotype of asexuality of the older demographic. 
Sexual Development of Older Adults

The first section of this paper focuses on the social elements that have lead to the current understanding of sexuality of older people. In this section, various developmental theories will be discussed in the context of how western society understands the development of sexuality in later life and how such theories have lead to the prominence of the asexual stereotype of older people (Sharpe, 2004). A review of literature pertaining to social factors of older people's lives that may act as barriers to sexual life will follow. Finally, media portrayals of sexuality of older people will be explored with their relation to the synthesis and perpetuation of notable stereotypes concerning sexuality of older adults. A review of new societal factors that effect the current older adult population will show how asexual stereotypes of those in later life stages are outdated and therefore no longer justifiable in modern times.

The second section reviews physical developments of aging during the later life stages and how these changes impact sexuality. Sex specific normative age-related changes like menopause and erectile dysfunction will be discussed in relation to their effect on sexual functioning. The consequence of age related illnesses on sexuality will be addressed, as well as the implications that normative changes and occurrence of illness have on societal perceptions of sexuality of older people. A review of physiological changes that impact sexual functioning will demonstrate the illogical nature of assuming asexuality is consequential to ageing.

The third section of this review centers on the psychological aspects of change in sexual functioning as well as psychological effects of the asexual stereotype on older people. Definitions of normative sexual aging will be discussed and the effect of these definitions on the emotions of older people experiencing these changes. The formation of 


\section{Kenny}

stereotypes based on societal perceptions (or misperceptions) of normative changes will be reviewed. Sexualities changing role in self-identity is explored, as well as the internalization of stereotypes in self-concept. Feelings of importance of sex are examined through a review of older people's conversations of reprioritization of sex in their lives due to a number of factors.

\section{Social}

A prominent stereotype of the elderly is that they are asexual. This stereotype manifests itself in society's negative reactions, such as disgust, shame and embarrassment to sexual expression of older people (Gott \& Hinchliff, 2003). Research has speculated that the root of the formation of the asexual older person stereotype is in the developmental theories of evolution and disengagement (Sharpe, 2004). Evolutionary theory suggests that sex is a functional act for the purpose of procreation, in this light; people not in the fertile period, such as the elderly, should not be seen as sexual. Many religious doctrines take traditional evolutionary ideas to the next level by deviantizing sex that is not for procreation, labeling it as a sin- hence, reinforcing asexual stereotype (Sharpe, 2004). Disengagement theory theorizes that society and individuals mutually withdraw from each other in later life. This theory reinforces many ageist views by suggesting that elderly people voluntarily and universally change their style of interaction from active to passive (Sharpe, 2004). Asexuality of older people is inferred from a disengagement theory viewpoint because asexuality is subsequent to a passive social role.

Other developmental theories support a less narrow view of sexuality and aging. For example, continuity theory suggests each individual deals with old age in much the 
Sexual Development of Older Adults

same way they coped with previous periods of life (Sharpe, 2004). Therefore, how one deals with changes in sexual life will vary depending on the person. Within this theory asexuality is not universally associated with aging. A theory with similar implications is Erikson's ego integrity theory, which speculates that a feeling of satisfaction in one's previous sexual experiences is the best predictor of a person's satisfaction with sexuality in later life (Sharpe, 2004). Continuity theory and ego integrity theory support views of sexuality and aging that do not foster negative stereotypes such as asexuality of older people. However, theoretical views such as evolutionary theory, that are ingrained in institutions like religion have greater influence on the point of view of the population because religion is integral to how many people understand the world. Therefore, due to the greater influence of theories that nourish asexual stereotypes of older people, asexual stereotypes persist.

Another social phenomenon that has great influence on creating and maintaining stereotypes is the media. Popular media reflects, creates and reinforces shared cultural ideas, and values (Decon, Minichiello, \& Plummer, 1995). Media such as television and movies have the power of displaying images of older people in a certain light, consequently transmitting specific messages and creating social meanings. Up until recently, it was taboo to display elderly people as sexualized in mainstream culture (Vares, 2009). Sexuality and old age were essentially understood as oppositional due to the prevalence of the asexual stereotype of older people. A new sexual image of older people was introduced to mainstream culture in the 1990s. This new image has been coined 'Sexy Oldie'. Sexy oldie is displayed in advertisements of performance enhancing drugs such as Viagra and Cialas (Vares, 2009). The fact that there are advertisements for 


\section{Kenny}

sexuo-pharmaceticals shows that there is a demand for such medication- this is evidence that the asexual stereotype is incorrect, or at the very least, not universally applicable (Hinchliff, 2009). A study set out to reveal the two extreme stereotypes as myths states that displaying elderly people as either sexy or asexual is restrictive. The sexy oldie stereotype pressures older people to conform to youthful guidelines of beauty and sex by taking performance-enhancing drugs (Hartzell, 2005) or under going cosmetic genitoplasty (which is increasingly popular in the United Kingdom) (Hinchliff, 2009). The asexual stereotype, on the other hand, has the potential to be internalized by older people, the result being older people coming to see sex at their age as deviant (Hartzell, 2005).

In a study done by Vares (2009), a sexually explicit scene from the movie, 'The Mother' displaying a normal looking older female was shown to groups of older people. The older people watching the film expressed feelings of 'unwatchability' of the scene, particularly the women. It was suggested by the subjects that despite the recent change in the way older people's sexuality is displayed in the media there has been a definite lifelong socialization to seeing only young female bodies as attractive (Vares, 2009).

There is a definite double standard in the way older males versus. older females are portrayed sexually in the media. Men are displayed as competent and sexual while women rarely are. This is evident in the frequent instances of older men being shown in romantic relationships with younger women and the infrequency of older women with younger men (Vares, 2009). Other research suggests this may be explained by evolutionary theory. Men are more likely to value youthfulness in a mate because it is a sign of fertility; in evolutionary theory human beings primary focus is on reproduction. 
Sexual Development of Older Adults

Males on the other hand continue to be fertile as they age, hence old age does not make men less attractive (Alterovits \& Mendelsohn, 2009). Media portrayals of older people are moving away from stereotypes of asexuality of older people by offering the opposite portrayal of sexy oldie as an alternative. However, ideas of asexuality of older people have long been ingrained in mainstream culture through social constructions of sexual attractiveness based on evolutionary values, so new oppositional ideas about the sexuality of older people- though they are truthful reflections of reality of some older people, are not easily incorporated into people's schemas about sexuality. Socially constructed ideas about sexuality and age are not only evident is media portrayals - normative changes in social life as people age contribute to mainstream understanding of sexuality and aging as well.

Certain social realities that accompany aging are thought, by some researchers, to be barriers to sexual life that lead to the perception of older people as asexual. These barriers include a decline in opportunity to establish relationships due to shrinking social networks that are a result of decreased mobility, death of friends, and retirement (Alterovits \& Mendelsohn, 2009). The increased likelihood that people, especially women, will be widowed as they age contributes to the assumption of asexuality in older people and reinforces the gender double standard (Hillman, 2008). Stereotypes of older people as asexual do not recognize that romantic relationships in later life are more and more common (Alterovits \& Mendelsohn, 2009).

Stereotypes of older adults as asexual persist because recent social trends that have been changing relationship structures over the past 50 years are not yet prevalent to mainstream culture. The social trends that are being referenced are the decline in 


\section{Kenny}

marriage rates and rise of divorce rates. As a result people, including the older population, now hold less strict beliefs about premarital sex (Hinchliff, 2009). A study analyzing older widows and widowers found that 18 months after the death of their spouse, $15 \%$ of women and $37 \%$ of men were interested in pursuing new romantic relationships (Alterovits \& Mendelsohn, 2009). Older adults are utilizing the Internet to overcome social barriers to forming new relationships. The Internet has allowed older people to easily access new social networks, where opportunities to find new romantic relationships are available (Alterovits \& Mendelsohn, 2009). It is clear that the romantic lifestyles of older adults are changing due to the changes in society such as less emphasis on wedlock as a necessary prelude to sexual relationships and introduction of instruments (the internet) that enable people to overcome social barriers to new relationships. It will take some time before the conventional image of older adult sexuality catches up to modern realities. To date, social constructions of ageing are much more relevant to perceptions of sexuality then physical elements of aging such as hormone changes and senescence of the sexual cycle (Hinchliff \& Gott, 2004).

\section{Physiological}

Older adult development is marked with normative changes in sexual functioning as well as increased prevalence of sexual dysfunction that is due to diseases associated with age. The normative sexual changes most commonly discussed in the literature on older adult development are menopause and erectile dysfunction. Common illnesses correlated with aging that effect sexual function include; heart disease, stroke, diabetes, depression and arthritis (Hillman, 2008). 
Sexual Development of Older Adults

The sexual cycle consists of desire, arousal, release and resolution. Both men and women experience senescence of the sexual cycle as they develop but to different extents. Arousal is the aspect of the sexual cycle that is most effected by age (Sharpe, 2004). In women this is due to the natural decreased levels of estrogen after menopause. Estrogen replacement therapy exists but it has been shown to be positively correlation with certain cancers, so it may be assumed that there are risks to this type of medication (Sharpe, 2004). For older men, the refractory period is especially affected, meaning they need a longer time to regroup after orgasm before they can achieve another orgasm. Women, on the other hand, tend not to have refractory phases. The orgasm phase is the phase that is least affected by aging. The arousal phase for both men and women is extended as they age; men in particular need a longer time of sustained stimulation for orgasm to occur. Women that have multiple orgasms can continue to experience this later in life, however some women experience pain with orgasm later in life due to orgasmic contractions becoming less coordinated with the body (Sharpe, 2004).

Menopause signifies the entrance into a new sexual stage of life for women. The symptoms of menopause vary greatly depending on the individual. The defining feature of menopause is the cessation of menstruation, which is accompanied by decreased and eventual ending of the production of estrogen. Lower levels of estrogen result in atrophy of urogenital tissues, decrease in lubrication during arousal and decreased clitoral size (Hillman, 2008). The results of lower estrogen levels as well as normative physical changes of shortening and narrowing of the vagina may lead to discomfort during intercourse (Sharpe, 2004). This discomfort may lead to the decrease in frequency of intercourse but desire, for both men and women, remains stable throughout life. The 


\section{Kenny}

decrease in frequency of intercourse may support the asexual stereotype of older adults but decrease in intercourse does not equate to decrease in sexual satisfaction; sex does not diminish as one ages, as much as it evolves (Sharpe, 2004).

For men, the most notable change in sexual function with age is erectile dysfunction. Erectile dysfunction commonly co-occurs with old age but it is not caused by aging per se (Sharpe, 2004). Just fewer than $40 \%$ of men experience some degree of erectile dysfunction by middle age and by age $70,67 \%$ of men experience moderate to severe erectile dysfunction (Hillman, 2008). Erectile dysfunction may increase in correlation with the development of illnesses across the life span.

For instance, erectile dysfunction is a potential side effect of diabetes because of decreased blood flow that results from diabetes. Other illnesses that are common with old age that can negatively affect sexual activities are heart disease, stroke, cancer, and arthritis. Arthritis for instance can cause pain during intercourse (Hillman, 2008). One study found that health disorders encountered in older age, like the ones listed above, have a greater impact on sexual functioning than the normative process of aging (Sharpe, 2004). Though aging may be correlated with decreased sexual function, asexuality is not consequential to the biological changes that occur with age nor is it necessarily consequential to the diseases that commonly occur in later life stages.

The asexual stereotype of older adults is further refuted by the instances of sexually transmitted diseases in the older adult population. Older adults account for $1.3 \%$ of all sexually transmitted diseases, this is due to the increased likelihood that older adults will practice unprotected sex because of the absence of the fear of pregnancy. Also, older women are more vulnerable to contracting sexually transmitted diseases than 
Sexual Development of Older Adults

younger women due to the increased likelihood of the tearing of the vaginal wall during intercourse, which allows viruses greater access to the blood stream (Hillman, 2008). A shocking statistic on new AIDS patients revealed that $12 \%$ to $20 \%$ of these patients are over 65 years old. The staggering amount of instances of sexually transmitted diseases in older people is partially due to limited knowledge of older adults to such risks. Older adults lacking knowledge about sexual health may be attributed to health care professionals assuming that older patients are generally asexual and therefore do not require knowledge about sexually transmitted diseases or sexual health assessments (Hillman, 2008). In this instance, the stereotype of asexuality poses as a real hazard for the well being of older adults. The perspectives of professionals need to be reoriented to better reflect the reality of modern sexual lifestyles of older people.

\section{Psychological}

The asexual stereotype of older adults can have potentially harmful psychological effects on older people, as it may cause distress, alter self-concept, and lead to the reprioritization of aspects of intimate relationships. Research has found that the interpretation of changes in sexual functioning being due to normative age related processes strongly impacts the amount of distress around these changes. Also, the amount of distress about how one is perceived sexually affects men and women differently as the value of sexuality in the context of identity varies depending on gender. Research has found that the importance of sex in later life is affected by societal prescriptions of appropriateness of sexuality in later life stages (Gott \& Hinchliff, 2003). 


\section{Kenny}

Older women experience being viewed as asexual negatively primarily because in a patriarchal society women hold sexual desirability as one of their main sources of power and influence over people (Deacon et al., 1995). Being viewed as asexual serves to strip women of their femininity, making older women disempowered (Vares, 2009). Sexuality is not only a source of power for women; it is also a defining feature of self because it is central to femininity and gender identity is central to self-identity. The physical changes associated with aging, such as loss of fertility and changes in appearance can constitute as failures of femininity, hence damaging a women's selfconcept (Vares, 2009). Asexual stereotypes are particularly harmful to women's psychological well being when they are internalized (Hillman, 2008).

Like women, when men internalize stereotypes of asexuality it can be harmful to their self-concept. When sexual dysfunction interferes with initiating sexual relations, a man's masculine identity may be damaged hence harming self-esteem. Men that are unaware of the normal physical changes of ageing may suffer from anxiety related to sexual performance. This anxiety about erectile dysfunction may become a self-fulfilling prophecy (Deacon et al., 1995).

Interviews with older adults in one study revealed that when barriers to sexual functioning were seen as part of the normal, natural aging process, distress concerning dysfunction decreased (Gott \& Hinchliff, 2003). This is especially true with cases of erectile dysfunction. Younger adults experiencing similar or milder degrees of erectile dysfunction were much more distressed then older adults that accepted it as natural (Hinchliff \& Gott, 2004). This is also applicable to the female process of menopause; some women going through menopause experience it as distressing because they perceive 
Sexual Development of Older Adults

the loss of reproductive capacity to be a signifier of loss of youth and therefore sexual desirability (Hinchliff \& Gott, 2004). Women who see menopause as an inevitable, natural aging process are found to benefit by actually enjoying sex more because they do not have to worry about the risk of pregnancy (Hinchliff \& Gott, 2011).

Acknowledgment of age as a causal factor in physical changes is seen to facilitate coping for both men and women and, in some cases, justifies the re-prioritization of sexual activity in life. In a study done by Gott and Hinchliff (2003), 44 participants aged 50 to 92 were asked to rate the importance of sex to their quality of life on the World Health Organization Quality of Life (WHOQOL) importance scale. The findings were split fairly evenly between three categories: not important, moderately important, and very important. The majority that indicated sex was not important said so because of health problems. Those that responded that sex was very important said so because it was an expression of love and of giving and receiving of pleasure. Sex to this group did not necessarily mean intercourse; sex could mean just cuddling. The defining feature of sex, to the 'very important' group was showing affection (Gott \& Hinchliff, 2003). In viewing the results it is understandable how the stereotype of asexuality of older people has arisen. However, a similar study, assessing only older married couples, found that only 2 out of 28 participants said sex was not important. So there is obviously a variety in the degree to which sex is thought to be of importance to older people, which may be effected by relationship status (Hinchliff \& Gott, 2011). However, an important factor of the asexual stereotype that has been overlooked is that there are a variety of definitions of sexuality that exist. Sexuality in the eyes of mainstream culture is centered on youth and copulation. While sexuality to an older person has an evolved definition that seems to be 


\section{Kenny}

focused on sharing spiritual connection and pleasure via some sort of physical communication (Hinchliff \& Gott, 2011). The prominent asexual stereotype of older people is based on a narrow definition of sexuality that does not take the grander scope of sexuality into account.

\section{Conclusion}

In conclusion, sexual stereotypes regarding older adults are based on societal assumptions about the aging process that are outdated; they persist because ideas that are ingrained in culture take time to change. Currently, the older demographic is much larger than before, and they face fewer social barriers to forming new relationships thanks to the innovations of the Internet. Also, the sheer size of the older adult cohort means there are more opportunities for new relationships. Physiological states of older people are also improving. For instance, more people are living to be older and have better health due to improvements in health care; this enables more and more older people to physiologically be able to maintain a sexual life. In modern times, social constructions of ageing have much more relevance to perceptions of sexuality then physical elements of aging (Hinchliff \& Gott, 2004). Research has shown that sexual desire is the stage of the sexual cycle that is least affected by senescence, so it is logical to assume that if older adults are physically able to have sex that more will take part in the activity (Sharpe, 2004). This shows that asexuality of older people is not a realistic assumption when there are no barriers to sexual expression.

In reviewing the research on psychological aspects of sexuality, it was found that the majority of older people that said they placed little importance on sexual relationships 


\section{Sexual Development of Older Adults}

did so because of health problems that restricted their ability to have sex (Gott \& Hinchliff, 2003). As explained previously, this does not support assumptions that asexuality is integral to aging. It may also be speculated that some older people internalize asexual stereotypes, which leads them to re-prioritize sex (Hillman, 2008).

Through reviewing the literature, it is obvious that the asexual stereotype is an over-generalized and outdated belief about the inherent qualities of sexuality in old age. The changing of stereotypes is a gradual process and the asexual stereotype is no exception. As new aspects of reality are digested by society, new social facts will emerge that show a different image of sexuality in later stages of life. 
Kenny

\section{References}

Alterovits, S., \& Mendelsohn, G. (2009). Partner preferences across the life span: Online dating by older adults. Psychology an Aging. 24(2), 513-517. doi:10.1037/a0015897

Deacon, S., Minichiello, V., \& Plummer, D. (1995). Sexuality and older people: Revisiting the assumptions. Educational Gerontology, 21(5), 497-513. doi:10.1080/0360127950210509

Gott, M., \& Hinchliff, S. (2003). How important is sex in later life?: The views of older people. Social Science \& Medicine, 56, 1617-1628.

Hartzell, R. (2005). Senior sex: Exploring the sex lives of older adults. [Review of the book Sexuality, sexual health, and ageing, by M. Gott]. The Journal of Sex Research, 43(3).

Hillman, J. (2008). Sexual issues and aging within the context of work. Professional Psychology: Research and Practice, 39(3), 290-297. doi: 10.1037/07357028.39.3.290

Hinchliff, S. (2009). Ageing and sexual health in the UK: How should health psychology respond to the challenges? Journal of Health Psychology, 14, 355-360. doi:10.1177/1359105308101673

Hinchliff, S., \& Gott, M. (2004). Perceptions of well-being in sexual ill health: What role does age play? Journal of Health Psychology, 9(5), 649-660. Doi:10.1177/1359105304045361

Hinchliff, S., \& Gott, M. (2004). Intimacy, commitment, and adaptation: Sexual relationships within long-term marriages. Journal of Social and Personal Relationships, 21 (5), 595-609. doi: 10.1177/0265407504045889

Sharpe, T. (2004). Introduction to sexuality in late life. The Family Journal, 12, 199-205. doi: $10.1177 / 002216780426410$

Vares, T. (2009). Reading the 'sexy oldie': Gender, age(ing) and embodiment. Sexualities, 12(4), 503-524. doi:10.1177/1363460709105716 